\title{
Turbulence: mechanics and structure of anomalous scaling
}

\author{
S. N. Gordienko ${ }^{1,2,3}$ and S. S. Moiseev ${ }^{1}$ \\ ${ }^{1}$ Space Research Institute, Profsoyuznaya str. 84/32, Moscow 117810, Russia \\ ${ }^{2}$ Max-Planck-Institut für Quantenoptik, 85748 Garching, Germany \\ ${ }^{3}$ Permanent address: L. D. Landau Institute of Theoretical Physics, 142432 Cherrogolovka, Moscow region, Russia
}

Received: 12 July 2000 - Revised: 18 December 2000 - Accepted: 20 February 2001

\begin{abstract}
As the finite correlation time of a force driving turbulence is taken into account, a new, dimensionless parameter occurs in the theory of turbulence. This new parameter is responsible for two different mechanisms of formation of anomalous spectra. The first mechanism is related to the change of a governing parameter, which defines the spectrum of turbulent fluctuation. The second mechanism is associated with spontaneous formation of characteristic scales that differ parametrically from the scale of the external force. The last mechanism can explain the intermittent structure of turbulent flows. The appropriate discrete set of the possible characteristic scales and anomalous spectra has been calculated. The results give a new insight into the concept of universality: there is a set of universal power laws, although occurrence in the spectrum segments described by one or another power law from this set depends on the dimensionless parameter mentioned above. It is noted that for the broad class of geophysical flows, the new dimensionless parameter is connected with the so-called degree of turbulence, which guarantees that the smallness of this parameter, as the degree of turbulence is usually small enough. That explains the important role of the Kolmogorov spectrum in geophysical applications.
\end{abstract}

\section{Introduction}

The transition of fluids or plasmas to a turbulent state is of fundamental importance for understanding the physics of a number of processes (including transport phenomena) taking place in these media. Yet, the best known example of strong turbulence is the turbulence in flows of incompressible fluids at high Reynolds numbers. The Kolmogorov spectrum and the concepts of universality, constant energy fluxes over spectrum, and the inertial range associated with it, are the basis of the modern understanding of the nature of homogeneous turbulence in incompressible fluids.

Correspondence to: S. S. Moiseev (moiseev@mx.iki.rssi.ru)
The main result of hydrodynamic turbulence is the Kolmogorov spectrum. It was derived more than fifty years ago and since then has been verified experimentally many times, but has never been obtained theoretically in an entirely satisfactory way. In other words, three main Kolmogorov assumptions have not been proven until now. As they are an important point of further consideration, we recall them: 1) the energy flow over the spectrum, which coincides with the dissipation rate, is the only important dimensional parameter describing turbulent fluctuations over the inertial interval, 2 ) the scale of a force driving turbulence is the only important scale of the theory and this scale determines the upper boundary of the inertial interval, 3) the dissipation rate is finite for vanishing viscosity. Let us look carefully at these three points.

First, the Kolmogorov spectrum, $E(k) \sim 1 / k^{5 / 3}$, is not the only spectrum of turbulent fluctuations that has been obtained experimentally in turbulent incompressible fluids. For instance, the spectrum $E(k) \sim 1 / k^{7 / 3}$ associated with the helicity flux is well known (Moiseev and Chkhetiani, 1996). It is, therefore, necessary A) to determine the conditions under which the rate of energy dissipation is a dimensional constant that determines the turbulent fluctuation spectrum and B) examine the question of how and under which conditions it is replaced by a dimensional parameter of a different character. It is interesting to recognize that the questions A) and B) have never been considered in the framework of the contemporary theory of turbulence. The current theory places its emphasis on a particular case, for which turbulence is driven by a force with the vanishing correlation time. Such a choice can be easily comprehended because it is the only case when the so-called Hopf or characteristic functional can be found in the closed form (Monin and Yaglom, 1967). Therefore, this case is especially convenient for theoretical investigation. Unfortunately the requirement of the theoretical convenience ignores a fact of physical importance. As one considers the case of the $\delta$-correlated force, the problem under consideration has only two dimensional parameters: the scale of the force driving turbulence and the 
parameter that has the dimension coinciding with the dimension of the dissipation rate of kinetic energy. In other words, in the contemporary microscopic theory of turbulence one has not considered the question why the complicated nonlinear dynamics of the Navier-Stokes equation forms a governing parameter with the dimension of the dissipation rate, but "forces" the inclusion of such a parameter in the statement of the problem. This approach refuses to consider the task concerning the dynamical roots of the dimensional parameter mentioned above, and reduces the problem of validity of the Kolmogorov theory to only the question about correlator dependence from the scale of a force driving turbulence. It is clear that this approach has limited applicability, and the finite correlation time of the force has to be taken into account.

Second, relations between the Kolmogorov spectrum and the so-called phenomenon of intermittency are not clear at the moment. It is generally accepted that intermittency means the occurrence of corrections to the Kolmogorov exponent, which equals $5 / 3$, and the main goal of a theory is to calculate these corrections. From this point of view, there are no differences between intermittency and the formation of different governing parameters under different types of the force driving incompressible flow. But this is not the case. The question is emphasized by the notion of a governing parameter, which can be considered for the model of homogeneous and uniform turbulence. At the same time, the most explicit experimental proof of intermittency comes from observations of the extremely inhomogeneous spatial and temporal distributions of small-scale turbulence, which vividly confirm the existence of the alternating structure of turbulent flows. This structure is exhibited as a tendency of small-scale turbulence and is concentrated in "bunches" surrounded by the area of the flow with a respectively low level of small-scale turbulent fluctuations (Monin and Yaglom, 1967). This experimental picture demonstrates that various structures which have different characteristic scales can occur in turbulent flows, and this is the experimental manifestation of intermittency. For this reason, the first question occurring in relation to the experimental picture described above is why new scales, which are parametrically distinguished from the scale of the external force driving an incompressible fluid, can arise, and what is the dimensionless parameter responsible for these new scales ("parametrically" is the key word in the sentence). Apparently, the origin of new scales means a radical modification of turbulent flows and results in the changing of the turbulent spectra.

From this point of view, the primary feature of intermittence is the origin of new, non-trivial scales in turbulent flows, rather than the "corrections" to the Kolmogorov spectrum, which represent only one of the consequences of the arising new scales. In other words, in a region of a turbulent flow, where a structure with the non-trivial scale exists, the turbulent spectrum evidently does not coincide with the Kolmogorov spectrum. The origin of a new scale is the key point for understanding of this phenomenon (to clarify this statement, notice that even the range of the vector numbers in which small-scale turbulence can be treated as homogeneous depends on the dimension of the "bunch", i.e. the characteristic scale of the given "bunch"). Therefore, the second Kolmogorov assumption should be revised. This point of view is consistent with experimental observations and allows us to distinguish clearly the essential difference between intermittency and the phenomenon of the changing of governing parameters, and has a simple physical meaning, namely that intermittency is connected with a spontaneous generation of structures in turbulent flows.

Third, one of the Kolmogorov assumptions is that the dissipation rate of kinetic energy does not depend on viscosity if viscosity goes to zero. For this reason, it is possible to ask what is the limit for the solutions of the Navier-Stokes equation that have the fixed dissipation rate when viscosity is vanishing? What is the equation describing the finite dissipation in the limit of vanishing viscosity? It was recognized long ago that the limit of the finite dissipation rate with vanishing viscosity corresponds to such solutions of the Euler equation, which do not have enough derivatives to allow integration by parts when one tries to prove energy conservation. The simplest way for rigorous mathematical treatment of such solutions is the "translation" of the problem into the language of Lagrangian particles. The special form of the Euler equation that can be regarded as the kinetic equation for Lagrangian particles and allows the mathematically rigorous studies of the finite dissipation with vanishing viscosity was obtained in Gordienko and Moiseev (1999). Such a formalism gives the opportunity to describe dynamics of an incompressible fluid by the equation, treating correctly and self-consistently the finite dissipation in the limit of the vanishing viscosity. The opportunity of such a treatment is quite obvious from physical reasonings, as it simply means that the small-scale dynamics near the viscous interval are guided by the large-scale motion and are determined by it. In this situation, any details of the "small-scale" dynamics are unimportant for large-scale behaviour of an incompressible fluid, and closed dissipative treatment of the large-scale dynamics is possible. It means that a closed description of finite dissipation for vanishing viscosity exists. The point of importance is that the kinetic equation mentioned above has the group of the scaling transformations which coincides with the group of the scaling transformations for the Euler equation. Namely, restoring the group of the scaling transformations corresponding to the Euler equation, together with the finite dissipation rate, is a point that determines most of the characteristics of turbulent flows.

The preceeding comments have clarified the purpose of the present work. We aim at taking into account the finite correlation time of a force driving turbulence. This consideration will give the opportunity to find out the domain of validity of the Kolmogorov theory if the dimensionless parameter, $\gamma$ (see below) is regarded properly. The only case of $3 D$-turbulence will be considered. 


\section{Types of governing parameters}

To describe turbulent flows driven by the external force $\boldsymbol{f}(\boldsymbol{r}, t)$, the Euler equation $(R e=+\infty)$ can be used (see the explanations above):

$$
\begin{aligned}
& \frac{\partial \boldsymbol{v}}{\partial t}+(\boldsymbol{v}, \nabla) \boldsymbol{v}=-\nabla p+\boldsymbol{f}(\boldsymbol{r}, t), \quad\langle\boldsymbol{f}(\boldsymbol{r}, t)\rangle=0 . \\
& \left\langle f_{\alpha}\left(\boldsymbol{r}_{1}, t_{1}\right) f_{\beta}\left(\boldsymbol{r}_{2}, t_{2}\right)\right\rangle=\delta_{\alpha \beta} f_{0}^{2} K\left(\frac{t_{1}-t_{2}}{\tau_{0}}, \frac{\boldsymbol{r}_{1}-\boldsymbol{r}_{2}}{r_{0}}\right) .
\end{aligned}
$$

It is pointed out once again that use of the Euler equation in such a problem means that its solutions of "low" smoothness are allowed. The mathematically correct treatment can be founded in Gordienko and Moiseev (1999). The physical meaning of these solutions is explained above. It is important to note that the group of the scaling transformation of the Euler equation, which is the basis for all results obtained below, gives the opportunity to derive them via a rigorous way, without any phenomenological assumptions (Gordienko and Moiseev, 1999). In this treatment of turbulence (it is sometimes called the Euler equation with the energy sink), the finite value of viscosity is only important if we are interested in the details of the dynamics near the viscous interval, but otherwise, it is completely unimportant for any other questions.

The function $K$ describes correlational properties of the force $f$ driving turbulence and according to the definition of $f_{0}^{2}$

$$
\int K\left(\frac{t}{\tau_{0}}, \frac{\boldsymbol{r}}{r_{0}}\right) d t d \boldsymbol{r}=r_{0}^{3} \tau_{0}
$$

where $r_{0}$ and $\tau_{0}$ are the correlational scale and time of the force. We are allowed to consider the force $f$ as a solenoidal one because we are able to include its potential part in the gradient of pressure. There are two dimensionless parameters $\gamma=f_{0} \tau_{0}^{2} / r_{0}, \Gamma=\gamma^{4 / 3} \operatorname{Re}$ and three dimensional parameters, $r_{0}, \tau_{0}, f_{0}$ in this problem.

At first, let us consider a change in the spectrum associated with the changing of a governing parameter, as the values of $\gamma$ and $\Gamma$ are increased. We mainly follow the results of Gordienko and Moiseev (1998) and Gordienko and Moiseev (1999), where additional details of mathematical character can be found.

If $\gamma \ll 1, \Gamma \ll 1$, the velocity correlator is

$\left\langle(\boldsymbol{v}(\boldsymbol{r}, t)-\boldsymbol{v}(0, t))^{2}\right\rangle=C_{1}\left(f_{0}^{2} \tau_{0} r\right)^{2 / 3}$

for $\lambda_{v} \ll r \ll r_{0}$, where $\lambda_{v}$ is the viscous length. Therefore, the Kolmogorov spectrum $E(k) \sim 1 / k^{5 / 3}$ is actually formed in the inertial interval.

Notice that the larger the force amplitude or the correlation time of the force, the larger the parameters $\gamma$ and $\Gamma$ are. The consequence of these parameters increasing is the change in the turbulent spectra. For $\gamma \ll 1, \Gamma \gg 1$, the new spectrum $E(k) \sim 1 / k^{2}$ arises near the viscous scale. This new spectrum cuts the Kolmogorov spectrum from the viscous range.
The velocity-velocity correlator is

$\left\langle(\boldsymbol{v}(\boldsymbol{r}, t)-\boldsymbol{v}(0, t))^{2}\right\rangle=C_{2}\left(f_{0} r\right)$

for $\lambda_{v} \ll r \ll \gamma r_{0}=f_{0} \tau_{0}^{2}$ and

$\left\langle(\boldsymbol{v}(\boldsymbol{r}, t)-\boldsymbol{v}(0, t))^{2}\right\rangle=C_{1}\left(f_{0}^{2} \tau_{0} r\right)^{2 / 3}$

for $\gamma r_{0}=f_{0} \tau_{0}^{2} \ll r \ll r_{0}$.

When the parameters $\gamma$ and $\Gamma$ reach the values $\gamma \gg 1$, $\Gamma \gg 1$, the whole inertial interval is occupied by this new spectrum $E(k) \sim 1 / k^{2}$.

A qualitatively new phenomenon occurs in long-scale domains, as the Kolmogorov spectrum is completely expelled from the inertial interval: the generation of large-scale structures with the characteristic scales reaching up to $\gamma^{2 / 5} r_{0}$ begins. It is reflected by an extremely slow decay of the velocity-velocity correlator beyond the inertial interval; actually

$\langle\boldsymbol{v}(\boldsymbol{r}, t) \boldsymbol{v}(0, t)\rangle=C_{3}\left(\frac{f_{0}^{2} r_{0}^{3}}{r}\right)^{1 / 2}$

for $r_{0} \ll r \ll \gamma^{2 / 5} r_{0}$.

Note that for the described way of the excitation of turbulence, the Loitsyansky's integral diverges for the arbitrary values of the parameters $\gamma$ and $\Gamma$. The long-scale part of the spectrum $k \ll \max \left(1 / r_{0}, \gamma^{2 / 5} / r_{0}\right)$ is universal and the spectrum is $E(k) \sim k^{1 / 3}$ in this realm of the wave-numbers. The last spectrum implies a slow decay of the velocity correlator:

$\langle\boldsymbol{v}(\boldsymbol{r}, t) \boldsymbol{v}(0, t)\rangle=C_{4}\left(\frac{f_{0}^{2} \tau_{0} r_{0}^{3}}{r^{2}}\right)^{2 / 3}$

for $r \gg \max \left(r_{0}, \gamma^{2 / 5} r_{0}\right)$. This unexpected theoretical result is consistent with the experimental dates (Eidelman et al., 2000).

The dissipation rate $\epsilon$ of kinetic energy can be estimated as the viscous losses in the scales of the order of magnitude of viscous length. It yields $\epsilon \sim f_{0}^{2} \tau_{0}$ for the case $\Gamma \ll 1$, i.e. the dissipation rate does not depend on viscosity. It is exactly the case of the Kolmogorov theory.

Let us make the similar estimation for $\gamma \ll 1, \Gamma \gg$ 1. For this case, the scale $f_{0} \tau_{0}^{2}$ exceeds the viscous length, i.e. the Kolmogorov spectrum is separated from the viscous range by the spectrum $E(k) \sim 1 / k^{2}$. The dissipation rate is $\epsilon \sim v^{1 / 3} f_{0}^{4 / 3}$. It is approximately $R e_{*}^{1 / 3}$ times less than the energy flux over the Kolmogorov segment of the spectrum, where $R e_{*}$ is the Reynolds number for the short wavelength end of the Kolmogorov spectrum and $v$ is the viscosity, respectively. Physical interpretation of this result was given in Gordienko and Moiseev (1998, 1999): the turbulent fluctuations that have the wave-number $k \sim 1 / r_{0}$ are in the so-called "spatial" resonance with an external force, and they are efficiently excited by the force. Then the energy moves over the Kolmogorov segment of the spectrum to the modes, that have a typical frequency $1 / \tau_{0}$ and are in "temporal" resonance with the external force. These modes 
oscillate in the counter-phase for the external force and the external force quenches their oscillations. In other words, the external force "takes" the energy back from the modes with $k \sim 1 / f_{0} \tau_{0}^{2}$. Therefore, an external force acts simultaneously as the source of energy and as the sink for the energy. This phenomenon of the collective sink of the energy is new physics associated with the finite correlation time of an external force.

\section{Intermittency in turbulent flows}

In the preceeding text, we have considered the mechanism of formation of anomalous spectra, which is related to the changing of the governing parameter for different values of $\gamma$ and $\Gamma$. It means that the dissipation rate of kinetic energy is not able to play the role of the governing parameter for the arbitrary correlation time of the external force. As it was pointed out in the beginning of the article, such a mechanism is not the only one. Other mechanisms of anomalous spectrum formation are connected with the violation of other Kolmogorov assumptions that allege that the characteristic scale of the turbulent flow is the scale of the external force. As we have a non-trivial dimensionless parameter $\gamma$, it is possible that the non-linear dynamics described by the NavierStokes equation (or, the so-called the Euler equation with the energy "sink") can spontaneously produce the characteristic scale which parametrically differs from the naive scale of the external force. We shall give a short review of results in this direction (see more details in Gordienko and Moiseev, 2001).

There is only a discrete set of possible characteristic scales that parametrically differ from the scale of the external force and the discrete set of the spectra corresponds to these scales. The appropriate velocity-velocity correlators are

$\left\langle(\boldsymbol{v}(\boldsymbol{r}, t)-\boldsymbol{v}(0, t))^{2}\right\rangle \sim r^{-2 \Delta(n)}$,

$\lambda_{v} \ll r \ll R(n)=r_{0} / \gamma^{1 /(n+1)}$

where $R(n)$ are new characteristic scales that differ parametrically from the scale $r_{0}$ of the external force, $n=1,2,3 \ldots$, $\Delta(n)=-(n+1) /(n+2)$. The spectrum of turbulent fluctuations corresponding to correlators (9) is $E_{n}(k) \sim k^{-1+2 \Delta(n)}$, $n=1,2,3 \ldots$

For the case $\gamma \ll 1 r_{0} \ll R(n)$, the largest characteristic scale corresponds to $n=1$. On the basis of dimensional arguments, the spectrum $E_{1}(k) \sim 1 / k^{7 / 3}$ can be interpreted as a spectrum formed by the finite helicity flux (Frish, 1973; Moffat, 1978; Moiseev and Chkhetiani, 1996).

The spontaneous generation of a new characteristic scale is interpreted as intermittency at the beginning of the article. These spectra exist only at those points of a turbulent flow where spontaneous formation of a non-trivial characteristic scale takes place. From this follows that intermittency reveals itself at the special regions of turbulent flows where spontaneous generation of a non-trivial scale has occurred. It is consistent with the observed picture of intermittency (Monin and Yaglom, 1967) and previous theoretical attempts to connect intermittency with spontaneous generation of intrinsically unstable long-range structures (Kuznetsov et. al, 1991).

The parameter $\gamma$ is not very convenient for geophysical applications. For this reason, it is necessary to emphasize that this parameter is very often connected with the so-called degree of turbulence. Degree of turbulence is defined as the ratio of the average square of the fluctuating component of the velocity to the square of the average velocity. There is no universal relationship between $\gamma$ and the degree of turbulence, although contemplation of particular cases corresponding to real geophysical flows proves that the less the degree of the turbulence is, the less the parameter $\gamma$ is. Moreover, the smallness of degree of turbulence causes the smallness of $\gamma$. Since the degree of turbulence is usually small, it clarifies the importance of the Kolmogorov theory for applications.

\section{References}

Belyan, A. V., Moiseev, S. S., and Chkhetiany, O. G., On turbulent viscosity in helical turbulence, Dokl. Akad. Nauk, 334(1), 3436, 1994.

Brissaud, A., Frisch, U., Leorat, J., Lessieur, M., and Mazure, A., Helicity cascades in fully developed isotropic turbulence, Phys. Fluids, 16, 1366-1367, 1973.

Eidelman, A., Branover, H., Gordienko, S., and Moiseev, S. S., Large-scale turbulence universality and the study of extreme weather events, Phys. Chem. Earth, 25, 35-38, 2000.

Gordienko, S. N. and Moiseev, S. S., Parametrization and universal properties of turbulence in incompressible fluids, JETP Letters, 68(3), 204-209, 1998.

Gordienko, S. N. and Moiseev, S. S., Structure of turbulent flows of incompressible fluids and parametrization of turbulence, JETP, 89(5), 880-889, 1999.

Gordienko, S. N. and Moiseev, S. S., "Internal" scale and large scale vortex formation, Int. J. Magnetohydrodynamics, in press, 2001.

Kuznetsov, E., Newell, A. C., and Zakharov, V. E., Intermittency and turbulence, Phys. Rev. Lett., 67, 3243, 1991.

Moffat, H. K., Magnetic Field Generation in Electrically Conducting Fluids, Cambridge Univ. Press, Cambridge, 1978.

Moiseev, S. and Chkhetiani, O. G., Helical scaling in turbulence, JETP, 83, 192-198, 1996.

Monin, A. S. and Yaglom, A. M., Statistical Hydromechanics, Part 2, Nauka, Moscow, in Russian, 1967. 